Acta Crystallographica Section E

Structure Reports

Online

ISSN 1600-5368

\section{(Z)-N-\{3-[(6-Chloropyridin-3-yl)methyl]- 1,3-thiazolidin-2-ylidene\}cyanamide}

\section{Jin-Sheng Gao, ${ }^{\mathrm{a}, \mathrm{b}_{*}}$ Jun Qiao, ${ }^{\mathrm{a}}$ Ying-Hui $\mathrm{Yu}^{\mathrm{a}}$ and Guang-Feng $\mathrm{Hou}^{\mathrm{b}}$}

${ }^{a}$ College of Chemistry and Materials Science, Heilongjiang University, Harbin 150080, People's Republic of China, and ${ }^{\mathbf{b}}$ Engineering Research Center of Pesticide of Heilongjiang Province, Heilongjiang University, Harbin 150080, People's Republic of China

Correspondence e-mail: hgf1000@163.com

Received 2 April 2011; accepted 8 April 2011

Key indicators: single-crystal X-ray study; $T=293 \mathrm{~K}$; mean $\sigma(\mathrm{C}-\mathrm{C})=0.004 \AA$; $R$ factor $=0.048 ; w R$ factor $=0.137 ;$ data-to-parameter ratio $=17.8$.

The asymmetric unit of the title compound, $\mathrm{C}_{10} \mathrm{H}_{9} \mathrm{ClN}_{4} \mathrm{~S}$, common name thiacloprid, comprises two molecules. In both molecules, the thiazolidine rings are almost planar (with r.m.s. deviations of 0.016 and $0.065 \AA$ ) and form dihedral angles of $73.36(6)$ and $70.25(8)^{\circ}$ with the 2-chloropyridine rings. In the crystal, intermolecular $\mathrm{C}-\mathrm{H} \cdots \mathrm{N}$ hydrogen bonds links the molecules into chains propagating in [101].

\section{Related literature}

For background to the title compound, a member of the neonicotinoide class of insecticides, see Maienfisch et al. (2003). For the synthesis, see Ishimitsu et al., (1991)

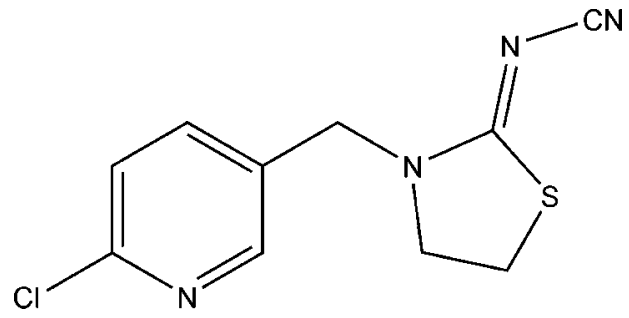

\section{Experimental}

Crystal data

$\mathrm{C}_{10} \mathrm{H}_{9} \mathrm{ClN}_{4} \mathrm{~S}$

$M_{r}=252.73$
Monoclinic, $P 2_{1} / c$ $a=7.1294$ (14) ̊

$$
\begin{aligned}
& b=35.469(7) \AA \\
& c=9.0211(18) \AA \\
& \beta=97.80(3)^{\circ} \\
& V=2260.1(8) \AA^{3} \\
& Z=8
\end{aligned}
$$

Data collection

Rigaku R-AXIS RAPID diffractometer

Absorption correction: multi-scan (ABSCOR; Higashi, 1995)

$T_{\min }=0.863, T_{\max }=0.909$

\section{Refinement}

$R\left[F^{2}>2 \sigma\left(F^{2}\right)\right]=0.048$

$w R\left(F^{2}\right)=0.137$

$S=1.08$

5151 reflections

Mo $K \alpha$ radiation

$\mu=0.50 \mathrm{~mm}^{-1}$

$T=293 \mathrm{~K}$

$0.31 \times 0.29 \times 0.20 \mathrm{~mm}$

21869 measured reflections 5151 independent reflections 3505 reflections with $I>2 \sigma(I)$ $R_{\text {int }}=0.046$

Table 1

Hydrogen-bond geometry $\left(\AA{ }^{\circ}\right)$.

\begin{tabular}{lllll}
\hline$D-\mathrm{H} \cdots A$ & $D-\mathrm{H}$ & $\mathrm{H} \cdots A$ & $D \cdots A$ & $D-\mathrm{H} \cdots A$ \\
\hline $\mathrm{C} 3-\mathrm{H} 3 \cdots \mathrm{N} 5^{\mathrm{i}}$ & 0.93 & 2.55 & $3.459(4)$ & 167 \\
$\mathrm{C} 13-\mathrm{H} 13 \cdots \mathrm{N} 1^{\mathrm{ii}}$ & 0.93 & 2.49 & $3.408(4)$ & 169 \\
\hline
\end{tabular}

Symmetry codes: (i) $x-1, y, z$; (ii) $x, y, z+1$.

Data collection: RAPID-AUTO (Rigaku, 1998); cell refinement: RAPID-AUTO; data reduction: CrystalClear (Rigaku/MSC, 2002); program(s) used to solve structure: SHELXS97 (Sheldrick, 2008); program(s) used to refine structure: SHELXL97 (Sheldrick, 2008); molecular graphics: SHELXTL (Sheldrick, 2008); software used to prepare material for publication: SHELXL97.

The authors thank the Special Funds for the Research of Scientific and Technological Innovative Talents of Harbin Municipal Science and Technology Bureau (2009RFXXG027), the Science and Technology Planning Project of Heilongjiang Province (GZ08A401) and Heilongjiang University for supporting this study.

Supplementary data and figures for this paper are available from the IUCr electronic archives (Reference: KP2319).

\section{References}

Higashi, T. (1995). ABSCOR. Rigaku Corporation, Tokyo, Japan.

Ishimitsu, K., Suzuki, J., Ohishi, H., Yamada, T., Hatano, R., Takakusa, N. \& Mitsui, J. (1991). WO Patent 91/04965.

Maienfisch, P., Haettenschwiler, J., Rindlisbacher, A., Decock, A., Wellmann, H. \& Kayser, H. (2003). Chimia, 57, 710-714.

Rigaku (1998). RAPID-AUTO. Rigaku Corporation, Tokyo, Japan.

Rigaku/MSC (2002). CrystalClear. Rigaku/MSC Inc., The Woodlands, Texas, USA.

Sheldrick, G. M. (2008). Acta Cryst. A64, 112-122. 


\section{supporting information}

Acta Cryst. (2011). E67, o1140 [doi:10.1107/S1600536811013316]

\section{(Z)-N-\{3-[(6-Chloropyridin-3-yl)methyl]-1,3-thiazolidin-2-ylidene\}cyanamide}

\section{Jin-Sheng Gao, Jun Qiao, Ying-Hui Yu and Guang-Feng Hou}

\section{S1. Comment}

Thiacloprid is the common name of the title compound, which is neonicotinoide class of insecticide. High efficacy and flexible application methods make it well suited for modern integrated pest management programs in many cropping systems (Ishimitsu et al., 1991; Maienfisch et al., 2003). We report here the synthesis and crystal structure of thiacloprid. The asymmetric unit comprises two molecules; the thiazolidine rings are almost planar, and form the dihedral angles with 2-chloropyridine rings of $73.36(6)$ and $70.25(8)^{\circ}$, respectively (Fig. 1).

In the crystal, the intermolecular $\mathrm{C}-\mathrm{H} \cdots \mathrm{N}$ hydrogen bonds link the molecules to form a chain (Fig. 2, Table 1).

\section{S2. Experimental}

The title compound was synthesised according the reference (Ishimitsu et al., 1991). A mixture of 2-cyanoiminothiazolidine (12.7 g, $0.1 \mathrm{~mol}$ ), 2-chloro-5-pyridylmethyl-chloride (17.4 g, $0.1 \mathrm{~mol})$, and $\mathrm{K}_{2} \mathrm{CO}_{3}(41.4 \mathrm{~g}, 0.3 \mathrm{~mol})$ in $150 \mathrm{~mL}$ of DMF was heated to $323 \mathrm{~K}$ and kept stirring for $7 \mathrm{~h}$. After filtered under reduced pressure, the DMF solution was distilled off. A total of $20.2 \mathrm{~g}(80.2 \%)$ title compound was obtained after the recrystallisation from ethyl acetate $(15 \mathrm{~mL})$. The suitable colourless block crystal was picked out for the single crystal $X$-ray diffaction measurement.

\section{S3. Refinement}

$\mathrm{H}$ atoms bound to $\mathrm{C}$ atoms were placed in calculated positions and treated as riding on their parent atoms, with $\mathrm{C}-\mathrm{H}=$ $0.93 \AA$ (aromatic); $\mathrm{C}-\mathrm{H}=0.97 \AA$ (methylene), and with $U_{\mathrm{iso}}(\mathrm{H})=1.2 U_{\mathrm{eq}}(\mathrm{C})$. 

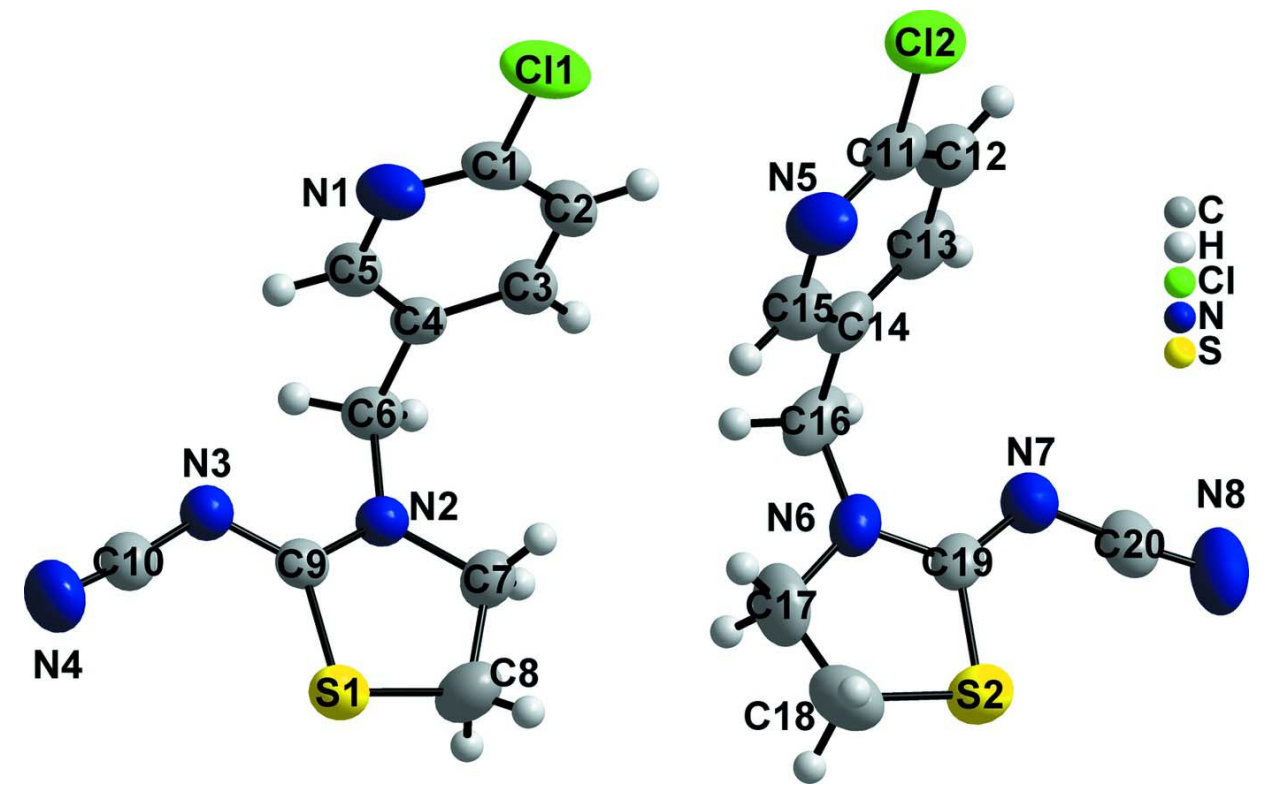

\section{Figure 1}

The molecular structure of the title compound showing displacement ellipsoids at the $50 \%$ probability level for non- $\mathrm{H}$ atoms.

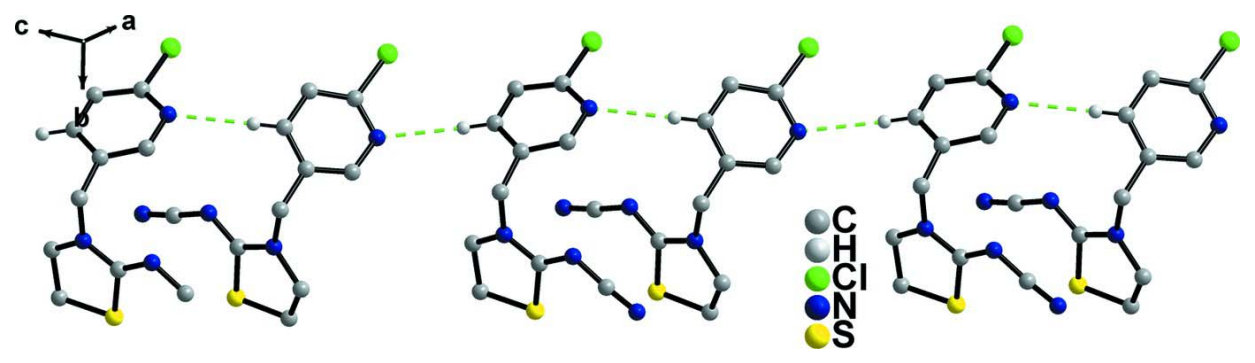

Figure 2

A partial packing view, showing the hydrogen bonding chain. Dashed lines indicate the hydrogen bonds, no involving $\mathrm{H}$ atoms have been omitted for clarity.

\section{(Z)-N-\{3-[(6-Chloropyridin-3-yl)methyl]- 1,3-thiazolidin-2-ylidene\}cyanamide}

Crystal data

$\mathrm{C}_{10} \mathrm{H}_{9} \mathrm{ClN}_{4} \mathrm{~S}$

$M_{r}=252.73$

Monoclinic, $P 2_{1} / c$

Hall symbol: -P $2 \mathrm{ybc}$

$a=7.1294$ (14) $\AA$

$b=35.469$ (7) $\AA$

$c=9.0211$ (18) $\AA$

$\beta=97.80(3)^{\circ}$

$V=2260.1(8) \AA^{3}$

$Z=8$
$F(000)=1040$

$D_{\mathrm{x}}=1.486 \mathrm{Mg} \mathrm{m}^{-3}$

Mo $K \alpha$ radiation, $\lambda=0.71073 \AA$

Cell parameters from 12991 reflections

$\theta=3.1-27.5^{\circ}$

$\mu=0.50 \mathrm{~mm}^{-1}$

$T=293 \mathrm{~K}$

Block, colourless

$0.31 \times 0.29 \times 0.20 \mathrm{~mm}$ 


\section{Data collection}

Rigaku R-AXIS RAPID diffractometer

Radiation source: fine-focus sealed tube Graphite monochromator $\omega$ scans

Absorption correction: multi-scan (ABSCOR; Higashi, 1995)

$T_{\min }=0.863, T_{\max }=0.909$

\section{Refinement}

Refinement on $F^{2}$

Least-squares matrix: full

$R\left[F^{2}>2 \sigma\left(F^{2}\right)\right]=0.048$

$w R\left(F^{2}\right)=0.137$

$S=1.08$

5151 reflections

289 parameters

0 restraints

Primary atom site location: structure-invariant direct methods
21869 measured reflections

5151 independent reflections

3505 reflections with $I>2 \sigma(I)$

$R_{\text {int }}=0.046$

$\theta_{\max }=27.5^{\circ}, \theta_{\min }=3.1^{\circ}$

$h=-8 \rightarrow 9$

$k=-45 \rightarrow 45$

$l=-11 \rightarrow 11$

Secondary atom site location: difference Fourier map

Hydrogen site location: inferred from neighbouring sites

$\mathrm{H}$-atom parameters constrained

$w=1 /\left[\sigma^{2}\left(F_{\mathrm{o}}^{2}\right)+(0.0595 P)^{2}+0.4995 P\right]$

where $P=\left(F_{\mathrm{o}}^{2}+2 F_{\mathrm{c}}^{2}\right) / 3$

$(\Delta / \sigma)_{\max }<0.001$

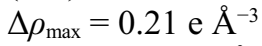

$\Delta \rho_{\min }=-0.29{\mathrm{e} \AA^{-3}}^{-3}$

Special details

Geometry. All e.s.d.'s (except the e.s.d. in the dihedral angle between two 1.s. planes) are estimated using the full covariance matrix. The cell e.s.d.'s are taken into account individually in the estimation of e.s.d.'s in distances, angles and torsion angles; correlations between e.s.d.'s in cell parameters are only used when they are defined by crystal symmetry. An approximate (isotropic) treatment of cell e.s.d.'s is used for estimating e.s.d.'s involving 1.s. planes.

Refinement. Refinement of $F^{2}$ against ALL reflections. The weighted $R$-factor $w R$ and goodness of fit $S$ are based on $F^{2}$, conventional $R$-factors $R$ are based on $F$, with $F$ set to zero for negative $F^{2}$. The threshold expression of $F^{2}>\sigma\left(F^{2}\right)$ is used only for calculating $R$-factors(gt) $e t c$. and is not relevant to the choice of reflections for refinement. $R$-factors based on $F^{2}$ are statistically about twice as large as those based on $F$, and $R$ - factors based on ALL data will be even larger.

Fractional atomic coordinates and isotropic or equivalent isotropic displacement parameters $\left(\AA^{2}\right)$

\begin{tabular}{lllll}
\hline & $x$ & $y$ & $z$ & $U_{\text {iso }} * / U_{\text {eq }}$ \\
\hline C1 & $0.8652(3)$ & $0.04805(6)$ & $0.2994(3)$ & $0.0528(6)$ \\
C2 & $0.7778(4)$ & $0.05431(7)$ & $0.4241(3)$ & $0.0564(6)$ \\
H2 & 0.8315 & 0.0461 & 0.5183 & $0.068^{*}$ \\
C3 & $0.6087(4)$ & $0.07309(7)$ & $0.4038(3)$ & $0.0541(6)$ \\
H3 & 0.5443 & 0.0777 & 0.4850 & $0.065^{*}$ \\
C4 & $0.5334(3)$ & $0.08520(5)$ & $0.2624(3)$ & $0.0417(5)$ \\
C5 & $0.6313(4)$ & $0.07628(6)$ & $0.1459(3)$ & $0.0525(6)$ \\
H5 & 0.5795 & 0.0835 & 0.0500 & $0.063^{*}$ \\
C6 & $0.3545(3)$ & $0.10860(6)$ & $0.2362(3)$ & $0.0486(5)$ \\
H6A & 0.2856 & 0.1025 & 0.1390 & $0.058^{*}$ \\
H6B & 0.2744 & 0.1024 & 0.3115 & $0.058^{*}$ \\
C7 & $0.4452(4)$ & $0.16785(7)$ & $0.3852(3)$ & $0.0586(6)$ \\
H7A & 0.5677 & 0.1592 & 0.4331 & $0.070^{*}$ \\
H7B & 0.3516 & 0.1622 & 0.4507 & $0.070^{*}$ \\
C8 & $0.4506(6)$ & $0.20899(8)$ & $0.3572(4)$ & $0.0831(10)$ \\
H8A & 0.5573 & 0.2202 & 0.4200 & $0.100^{*}$
\end{tabular}




$\begin{array}{lllll}\text { H8B } & 0.3354 & 0.2207 & 0.3811 & 0.100^{*} \\ \text { C9 } & 0.4189(3) & 0.16902(6) & 0.1222(3) & 0.0422(5) \\ \text { C10 } & 0.4177(3) & 0.17589(7) & -0.1287(3) & 0.0545(6) \\ \text { C11 } & 1.3337(3) & 0.05498(7) & 0.7709(3) & 0.0558(6) \\ \text { C12 } & 1.2138(4) & 0.04665(8) & 0.8736(3) & 0.0618(6) \\ \text { H12 } & 1.2387 & 0.0268 & 0.9406 & 0.074^{*} \\ \text { C13 } & 1.0549(4) & 0.06898(8) & 0.8737(3) & 0.0609(7) \\ \text { H13 } & 0.9712 & 0.0646 & 0.9423 & 0.073^{*} \\ \text { C14 } & 1.0214(3) & 0.09783(7) & 0.7712(3) & 0.0523(6) \\ \text { C15 } & 1.1509(4) & 0.10300(8) & 0.6735(3) & 0.0665(7) \\ \text { H15 } & 1.1280 & 0.1222 & 0.6033 & 0.080^{*} \\ \text { C16 } & 0.8483(3) & 0.12283(9) & 0.7672(4) & 0.0678(8) \\ \text { H16A } & 0.7818 & 0.1234 & 0.6660 & 0.081^{*} \\ \text { H16B } & 0.7636 & 0.1121 & 0.8315 & 0.081^{*} \\ \text { C17 } & 0.8939(5) & 0.19245(10) & 0.7102(4) & 0.0840(10) \\ \text { H17A } & 0.7664 & 0.1963 & 0.6595 & 0.101^{*} \\ \text { H17B } & 0.9753 & 0.1866 & 0.6353 & 0.101^{*} \\ \text { C18 } & 0.9605(6) & 0.22673(9) & 0.7904(4) & 0.0853(10) \\ \text { H18A } & 0.8799 & 0.2478 & 0.7556 & 0.102^{*} \\ \text { H18B } & 1.0887 & 0.2325 & 0.7728 & 0.102^{*} \\ \text { C19 } & 0.9155(3) & 0.17075(7) & 0.9595(3) & 0.0464(5) \\ \text { C20 } & 0.9267(3) & 0.15741(8) & 1.2059(3) & 0.0593(6) \\ \text { C11 } & 1.08715(10) & 0.02601(2) & 0.31970(11) & 0.0811(3) \\ \text { C12 } & 1.53924(11) & 0.02813(2) & 0.76846(10) & 0.0809(2) \\ \text { N1 } & 0.7967(3) & 0.05776(6) & 0.1620(3) & 0.0592(5) \\ \text { N2 } & 0.3963(2) & 0.14899(5) & 0.2425(2) & 0.0432(4) \\ \text { N3 } & 0.4000(3) & 0.15412(5) & -0.0121(2) & 0.0504(5) \\ \text { N4 } & 0.4288(4) & 0.19221(7) & -0.2375(3) & 0.0777(7) \\ \text { N5 } & 1.3080(3) & 0.08234(7) & 0.6720(3) & 0.0658(6) \\ \text { N6 } & 0.8957(3) & 0.16118(6) & 0.8155(2) & 0.0540(5) \\ \text { N7 } & 0.9051(3) & 0.14604(6) & 1.0652(2) & 0.0551(5) \\ \text { N8 } & 0.9439(4) & 0.16423(9) & 1.3319(3) & 0.0864(8) \\ \text { S1 } & 0.47264(10) & 0.216369(16) & 0.16494(8) & 0.05802(19) \\ \text { S2 } & 0.95422(12) & 0.21907(2) & 0.98475(9) & 0.0698(2) \\ & & & & \end{array}$

Atomic displacement parameters $\left(\AA^{2}\right)$

\begin{tabular}{lllllll}
\hline & $U^{11}$ & $U^{22}$ & $U^{33}$ & $U^{12}$ & $U^{13}$ & $U^{23}$ \\
\hline C1 & $0.0496(12)$ & $0.0361(10)$ & $0.0750(18)$ & $0.0031(10)$ & $0.0159(12)$ & $0.0066(11)$ \\
C2 & $0.0653(14)$ & $0.0536(13)$ & $0.0499(15)$ & $0.0087(12)$ & $0.0069(12)$ & $0.0066(11)$ \\
C3 & $0.0656(14)$ & $0.0505(12)$ & $0.0483(14)$ & $0.0063(11)$ & $0.0159(11)$ & $0.0021(11)$ \\
C4 & $0.0476(11)$ & $0.0336(9)$ & $0.0458(13)$ & $-0.0015(9)$ & $0.0138(9)$ & $0.0020(8)$ \\
C5 & $0.0664(14)$ & $0.0486(12)$ & $0.0450(14)$ & $0.0081(11)$ & $0.0160(11)$ & $0.0053(10)$ \\
C6 & $0.0465(11)$ & $0.0424(11)$ & $0.0580(15)$ & $-0.0045(9)$ & $0.0113(10)$ & $0.0002(10)$ \\
C7 & $0.0753(16)$ & $0.0539(13)$ & $0.0462(14)$ & $-0.0019(12)$ & $0.0061(12)$ & $-0.0049(11)$ \\
C8 & $0.133(3)$ & $0.0574(16)$ & $0.060(2)$ & $0.0008(18)$ & $0.0185(19)$ & $-0.0117(14)$ \\
C9 & $0.0367(10)$ & $0.0416(10)$ & $0.0476(13)$ & $0.0033(8)$ & $0.0029(9)$ & $0.0007(9)$ \\
C10 & $0.0555(13)$ & $0.0566(13)$ & $0.0501(15)$ & $0.0046(11)$ & $0.0032(11)$ & $0.0001(12)$
\end{tabular}


supporting information

\begin{tabular}{lllllll} 
C11 & $0.0568(13)$ & $0.0556(13)$ & $0.0579(16)$ & $-0.0027(11)$ & $0.0177(12)$ & $-0.0152(12)$ \\
C12 & $0.0708(16)$ & $0.0605(15)$ & $0.0575(17)$ & $-0.0074(13)$ & $0.0214(13)$ & $-0.0025(12)$ \\
C13 & $0.0601(14)$ & $0.0736(16)$ & $0.0541(16)$ & $-0.0178(13)$ & $0.0257(12)$ & $-0.0177(13)$ \\
C14 & $0.0495(12)$ & $0.0619(14)$ & $0.0465(14)$ & $-0.0090(11)$ & $0.0106(10)$ & $-0.0205(11)$ \\
C15 & $0.0728(17)$ & $0.0727(17)$ & $0.0591(18)$ & $0.0088(14)$ & $0.0279(14)$ & $0.0006(13)$ \\
C16 & $0.0482(13)$ & $0.0861(19)$ & $0.0685(19)$ & $-0.0033(13)$ & $0.0062(12)$ & $-0.0297(15)$ \\
C17 & $0.096(2)$ & $0.112(3)$ & $0.0459(17)$ & $0.009(2)$ & $0.0141(15)$ & $0.0145(17)$ \\
C18 & $0.106(2)$ & $0.0733(19)$ & $0.080(2)$ & $0.0222(18)$ & $0.0239(19)$ & $0.0220(17)$ \\
C19 & $0.0389(10)$ & $0.0567(13)$ & $0.0438(13)$ & $0.0051(10)$ & $0.0068(9)$ & $-0.0056(10)$ \\
C20 & $0.0484(12)$ & $0.0786(17)$ & $0.0525(16)$ & $0.0114(12)$ & $0.0125(11)$ & $0.0098(13)$ \\
C11 & $0.0568(4)$ & $0.0582(4)$ & $0.1311(8)$ & $0.0141(3)$ & $0.0232(4)$ & $0.0113(4)$ \\
C12 & $0.0785(5)$ & $0.0756(5)$ & $0.0938(6)$ & $0.0180(4)$ & $0.0307(4)$ & $-0.0044(4)$ \\
N1 & $0.0684(13)$ & $0.0531(11)$ & $0.0623(15)$ & $0.0100(10)$ & $0.0310(11)$ & $0.0084(10)$ \\
N2 & $0.0454(9)$ & $0.0407(9)$ & $0.0437(11)$ & $0.0020(8)$ & $0.0068(8)$ & $-0.0008(8)$ \\
N3 & $0.0559(11)$ & $0.0496(10)$ & $0.0450(12)$ & $0.0029(9)$ & $0.0045(9)$ & $-0.0002(9)$ \\
N4 & $0.0968(18)$ & $0.0831(17)$ & $0.0537(15)$ & $0.0063(14)$ & $0.0119(13)$ & $0.0126(13)$ \\
N5 & $0.0701(14)$ & $0.0680(13)$ & $0.0662(15)$ & $0.0064(11)$ & $0.0349(12)$ & $-0.0018(11)$ \\
N6 & $0.0504(10)$ & $0.0702(13)$ & $0.0413(11)$ & $0.0095(10)$ & $0.0058(8)$ & $-0.0082(9)$ \\
N7 & $0.0581(11)$ & $0.0601(12)$ & $0.0485(13)$ & $0.0040(10)$ & $0.0129(9)$ & $0.0025(9)$ \\
N8 & $0.0813(17)$ & $0.133(2)$ & $0.0470(15)$ & $0.0174(16)$ & $0.0147(12)$ & $0.0044(15)$ \\
S1 & $0.0681(4)$ & $0.0420(3)$ & $0.0615(4)$ & $-0.0052(3)$ & $-0.0003(3)$ & $0.0019(3)$ \\
S2 & $0.0892(5)$ & $0.0566(4)$ & $0.0649(5)$ & $0.0004(3)$ & $0.0145(4)$ & $-0.0053(3)$ \\
& & & & & & \\
\hline
\end{tabular}

Geometric parameters $\left(\AA,{ }^{\circ}\right)$

\begin{tabular}{llll}
\hline $\mathrm{C} 1-\mathrm{N} 1$ & $1.314(3)$ & $\mathrm{C} 11-\mathrm{N} 5$ & $1.314(4)$ \\
$\mathrm{C} 1-\mathrm{C} 2$ & $1.376(4)$ & $\mathrm{C} 11-\mathrm{C} 12$ & $1.375(4)$ \\
$\mathrm{C} 1-\mathrm{C} 11$ & $1.752(2)$ & $\mathrm{C} 11-\mathrm{C} 2$ & $1.750(3)$ \\
$\mathrm{C} 2-\mathrm{C} 3$ & $1.368(3)$ & $\mathrm{C} 12-\mathrm{C} 13$ & $1.383(4)$ \\
$\mathrm{C} 2-\mathrm{H} 2$ & 0.9300 & $\mathrm{C} 12-\mathrm{H} 12$ & 0.9300 \\
$\mathrm{C} 3-\mathrm{C} 4$ & $1.383(3)$ & $\mathrm{C} 13-\mathrm{C} 14$ & $1.378(4)$ \\
$\mathrm{C} 3-\mathrm{H} 3$ & 0.9300 & $\mathrm{C} 13-\mathrm{H} 13$ & 0.9300 \\
$\mathrm{C} 4-\mathrm{C} 5$ & $1.375(3)$ & $\mathrm{C} 14-\mathrm{C} 15$ & $1.373(4)$ \\
$\mathrm{C} 4-\mathrm{C} 6$ & $1.513(3)$ & $\mathrm{C} 14-\mathrm{C} 16$ & $1.516(4)$ \\
$\mathrm{C} 5-\mathrm{N} 1$ & $1.340(3)$ & $\mathrm{C} 15-\mathrm{N} 5$ & $1.340(4)$ \\
$\mathrm{C} 5-\mathrm{H} 5$ & 0.9300 & $\mathrm{C} 15-\mathrm{H} 15$ & 0.9300 \\
$\mathrm{C} 6-\mathrm{N} 2$ & $1.463(3)$ & $\mathrm{C} 16-\mathrm{N} 6$ & $1.454(3)$ \\
$\mathrm{C} 6-\mathrm{H} 6 \mathrm{~A}$ & 0.9700 & $\mathrm{C} 16-\mathrm{H} 16 \mathrm{~A}$ & 0.9700 \\
$\mathrm{C} 6-\mathrm{H} 6 \mathrm{~B}$ & 0.9700 & $\mathrm{C} 16-\mathrm{H} 16 \mathrm{~B}$ & 0.9700 \\
$\mathrm{C} 7-\mathrm{N} 2$ & $1.451(3)$ & $\mathrm{C} 17-\mathrm{N} 6$ & $1.459(4)$ \\
$\mathrm{C} 7-\mathrm{C} 8$ & $1.482(4)$ & $\mathrm{C} 17-\mathrm{C} 18$ & $1.461(5)$ \\
$\mathrm{C} 7-\mathrm{H} 7 \mathrm{~A}$ & 0.9700 & $\mathrm{C} 17-\mathrm{H} 17 \mathrm{~A}$ & 0.9700 \\
$\mathrm{C} 7-\mathrm{H} 7 \mathrm{~B}$ & 0.9700 & $\mathrm{C} 17-\mathrm{H} 17 \mathrm{~B}$ & 0.9700 \\
$\mathrm{C} 8-\mathrm{S} 1$ & $1.782(3)$ & $\mathrm{C} 18-\mathrm{S} 2$ & $1.781(4)$ \\
$\mathrm{C} 8-\mathrm{H} 8 \mathrm{~A}$ & 0.9700 & $\mathrm{C} 18-\mathrm{H} 18 \mathrm{~A}$ & 0.9700 \\
$\mathrm{C} 8-\mathrm{H} 8 \mathrm{~B}$ & 0.9700 & $\mathrm{C} 18-\mathrm{H} 18 \mathrm{~B}$ & 0.9700 \\
$\mathrm{C} 9-\mathrm{N} 3$ & $1.312(3)$ & $\mathrm{C} 19-\mathrm{N} 7$ & $1.305(3)$ \\
$\mathrm{C} 9-\mathrm{N} 2$ & $1.325(3)$ & $\mathrm{C} 19-\mathrm{N} 6$ & $1.331(3)$ \\
& & &
\end{tabular}




\begin{tabular}{|c|c|c|c|}
\hline $\mathrm{C} 9-\mathrm{S} 1$ & $1.754(2)$ & $\mathrm{C} 19-\mathrm{S} 2$ & $1.746(2)$ \\
\hline $\mathrm{C} 10-\mathrm{N} 4$ & $1.152(3)$ & $\mathrm{C} 20-\mathrm{N} 8$ & $1.152(4)$ \\
\hline $\mathrm{C} 10-\mathrm{N} 3$ & $1.324(3)$ & $\mathrm{C} 20-\mathrm{N} 7$ & $1.321(3)$ \\
\hline $\mathrm{N} 1-\mathrm{C} 1-\mathrm{C} 2$ & $125.2(2)$ & $\mathrm{C} 14-\mathrm{C} 13-\mathrm{C} 12$ & $119.5(2)$ \\
\hline $\mathrm{N} 1-\mathrm{C} 1-\mathrm{Cl} 1$ & $115.5(2)$ & $\mathrm{C} 14-\mathrm{C} 13-\mathrm{H} 13$ & 120.2 \\
\hline $\mathrm{C} 2-\mathrm{C} 1-\mathrm{Cl} 1$ & $119.3(2)$ & $\mathrm{C} 12-\mathrm{C} 13-\mathrm{H} 13$ & 120.2 \\
\hline $\mathrm{C} 3-\mathrm{C} 2-\mathrm{C} 1$ & $117.2(2)$ & $\mathrm{C} 15-\mathrm{C} 14-\mathrm{C} 13$ & $117.3(2)$ \\
\hline $\mathrm{C} 3-\mathrm{C} 2-\mathrm{H} 2$ & 121.4 & $\mathrm{C} 15-\mathrm{C} 14-\mathrm{C} 16$ & $121.5(3)$ \\
\hline $\mathrm{C} 1-\mathrm{C} 2-\mathrm{H} 2$ & 121.4 & $\mathrm{C} 13-\mathrm{C} 14-\mathrm{C} 16$ & $121.1(3)$ \\
\hline $\mathrm{C} 2-\mathrm{C} 3-\mathrm{C} 4$ & $120.0(2)$ & N5-C15-C14 & $124.7(3)$ \\
\hline $\mathrm{C} 2-\mathrm{C} 3-\mathrm{H} 3$ & 120.0 & $\mathrm{~N} 5-\mathrm{C} 15-\mathrm{H} 15$ & 117.6 \\
\hline $\mathrm{C} 4-\mathrm{C} 3-\mathrm{H} 3$ & 120.0 & $\mathrm{C} 14-\mathrm{C} 15-\mathrm{H} 15$ & 117.6 \\
\hline $\mathrm{C} 5-\mathrm{C} 4-\mathrm{C} 3$ & $117.4(2)$ & $\mathrm{N} 6-\mathrm{C} 16-\mathrm{C} 14$ & $112.62(19)$ \\
\hline $\mathrm{C} 5-\mathrm{C} 4-\mathrm{C} 6$ & $120.7(2)$ & $\mathrm{N} 6-\mathrm{C} 16-\mathrm{H} 16 \mathrm{~A}$ & 109.1 \\
\hline $\mathrm{C} 3-\mathrm{C} 4-\mathrm{C} 6$ & $121.9(2)$ & $\mathrm{C} 14-\mathrm{C} 16-\mathrm{H} 16 \mathrm{~A}$ & 109.1 \\
\hline $\mathrm{N} 1-\mathrm{C} 5-\mathrm{C} 4$ & $124.1(2)$ & N6-C16-H16B & 109.1 \\
\hline $\mathrm{N} 1-\mathrm{C} 5-\mathrm{H} 5$ & 118.0 & $\mathrm{C} 14-\mathrm{C} 16-\mathrm{H} 16 \mathrm{~B}$ & 109.1 \\
\hline $\mathrm{C} 4-\mathrm{C} 5-\mathrm{H} 5$ & 118.0 & $\mathrm{H} 16 \mathrm{~A}-\mathrm{C} 16-\mathrm{H} 16 \mathrm{~B}$ & 107.8 \\
\hline $\mathrm{N} 2-\mathrm{C} 6-\mathrm{C} 4$ & $111.60(17)$ & $\mathrm{N} 6-\mathrm{C} 17-\mathrm{C} 18$ & $109.7(3)$ \\
\hline $\mathrm{N} 2-\mathrm{C} 6-\mathrm{H} 6 \mathrm{~A}$ & 109.3 & $\mathrm{~N} 6-\mathrm{C} 17-\mathrm{H} 17 \mathrm{~A}$ & 109.7 \\
\hline $\mathrm{C} 4-\mathrm{C} 6-\mathrm{H} 6 \mathrm{~A}$ & 109.3 & $\mathrm{C} 18-\mathrm{C} 17-\mathrm{H} 17 \mathrm{~A}$ & 109.7 \\
\hline $\mathrm{N} 2-\mathrm{C} 6-\mathrm{H} 6 \mathrm{~B}$ & 109.3 & N6-C17-H17B & 109.7 \\
\hline $\mathrm{C} 4-\mathrm{C} 6-\mathrm{H} 6 \mathrm{~B}$ & 109.3 & $\mathrm{C} 18-\mathrm{C} 17-\mathrm{H} 17 \mathrm{~B}$ & 109.7 \\
\hline $\mathrm{H} 6 \mathrm{~A}-\mathrm{C} 6-\mathrm{H} 6 \mathrm{~B}$ & 108.0 & $\mathrm{H} 17 \mathrm{~A}-\mathrm{C} 17-\mathrm{H} 17 \mathrm{~B}$ & 108.2 \\
\hline $\mathrm{N} 2-\mathrm{C} 7-\mathrm{C} 8$ & $108.2(2)$ & $\mathrm{C} 17-\mathrm{C} 18-\mathrm{S} 2$ & $108.1(2)$ \\
\hline $\mathrm{N} 2-\mathrm{C} 7-\mathrm{H} 7 \mathrm{~A}$ & 110.1 & $\mathrm{C} 17-\mathrm{C} 18-\mathrm{H} 18 \mathrm{~A}$ & 110.1 \\
\hline $\mathrm{C} 8-\mathrm{C} 7-\mathrm{H} 7 \mathrm{~A}$ & 110.1 & $\mathrm{~S} 2-\mathrm{C} 18-\mathrm{H} 18 \mathrm{~A}$ & 110.1 \\
\hline $\mathrm{N} 2-\mathrm{C} 7-\mathrm{H} 7 \mathrm{~B}$ & 110.1 & $\mathrm{C} 17-\mathrm{C} 18-\mathrm{H} 18 \mathrm{~B}$ & 110.1 \\
\hline $\mathrm{C} 8-\mathrm{C} 7-\mathrm{H} 7 \mathrm{~B}$ & 110.1 & $\mathrm{~S} 2-\mathrm{C} 18-\mathrm{H} 18 \mathrm{~B}$ & 110.1 \\
\hline $\mathrm{H} 7 \mathrm{~A}-\mathrm{C} 7-\mathrm{H} 7 \mathrm{~B}$ & 108.4 & $\mathrm{H} 18 \mathrm{~A}-\mathrm{C} 18-\mathrm{H} 18 \mathrm{~B}$ & 108.4 \\
\hline $\mathrm{C} 7-\mathrm{C} 8-\mathrm{S} 1$ & $108.5(2)$ & $\mathrm{N} 7-\mathrm{C} 19-\mathrm{N} 6$ & $122.2(2)$ \\
\hline $\mathrm{C} 7-\mathrm{C} 8-\mathrm{H} 8 \mathrm{~A}$ & 110.0 & $\mathrm{~N} 7-\mathrm{C} 19-\mathrm{S} 2$ & $125.99(19)$ \\
\hline $\mathrm{S} 1-\mathrm{C} 8-\mathrm{H} 8 \mathrm{~A}$ & 110.0 & $\mathrm{~N} 6-\mathrm{C} 19-\mathrm{S} 2$ & $111.81(18)$ \\
\hline $\mathrm{C} 7-\mathrm{C} 8-\mathrm{H} 8 \mathrm{~B}$ & 110.0 & $\mathrm{~N} 8-\mathrm{C} 20-\mathrm{N} 7$ & $174.3(3)$ \\
\hline $\mathrm{S} 1-\mathrm{C} 8-\mathrm{H} 8 \mathrm{~B}$ & 110.0 & $\mathrm{C} 1-\mathrm{N} 1-\mathrm{C} 5$ & $116.1(2)$ \\
\hline $\mathrm{H} 8 \mathrm{~A}-\mathrm{C} 8-\mathrm{H} 8 \mathrm{~B}$ & 108.4 & $\mathrm{C} 9-\mathrm{N} 2-\mathrm{C} 7$ & $116.01(19)$ \\
\hline N3-C9-N2 & $122.1(2)$ & $\mathrm{C} 9-\mathrm{N} 2-\mathrm{C} 6$ & $122.68(19)$ \\
\hline $\mathrm{N} 3-\mathrm{C} 9-\mathrm{S} 1$ & $125.52(18)$ & $\mathrm{C} 7-\mathrm{N} 2-\mathrm{C} 6$ & $120.61(19)$ \\
\hline $\mathrm{N} 2-\mathrm{C} 9-\mathrm{S} 1$ & $112.34(17)$ & $\mathrm{C} 9-\mathrm{N} 3-\mathrm{C} 10$ & $119.3(2)$ \\
\hline $\mathrm{N} 4-\mathrm{C} 10-\mathrm{N} 3$ & $174.2(3)$ & $\mathrm{C} 11-\mathrm{N} 5-\mathrm{C} 15$ & $115.9(2)$ \\
\hline $\mathrm{N} 5-\mathrm{C} 11-\mathrm{C} 12$ & $125.0(2)$ & $\mathrm{C} 19-\mathrm{N} 6-\mathrm{C} 16$ & $121.3(2)$ \\
\hline $\mathrm{N} 5-\mathrm{C} 11-\mathrm{Cl} 2$ & $115.7(2)$ & $\mathrm{C} 19-\mathrm{N} 6-\mathrm{C} 17$ & $115.6(2)$ \\
\hline $\mathrm{C} 12-\mathrm{C} 11-\mathrm{Cl} 2$ & $119.3(2)$ & $\mathrm{C} 16-\mathrm{N} 6-\mathrm{C} 17$ & $122.3(2)$ \\
\hline $\mathrm{C} 11-\mathrm{C} 12-\mathrm{C} 13$ & $117.5(3)$ & $\mathrm{C} 19-\mathrm{N} 7-\mathrm{C} 20$ & $119.1(2)$ \\
\hline $\mathrm{C} 11-\mathrm{C} 12-\mathrm{H} 12$ & 121.3 & $\mathrm{C} 9-\mathrm{S} 1-\mathrm{C} 8$ & $91.61(12)$ \\
\hline $\mathrm{C} 13-\mathrm{C} 12-\mathrm{H} 12$ & 121.3 & $\mathrm{C} 19-\mathrm{S} 2-\mathrm{C} 18$ & $92.62(14)$ \\
\hline
\end{tabular}




\section{supporting information}

Hydrogen-bond geometry $\left(A,{ }^{\circ}\right)$

\begin{tabular}{lllll}
\hline$D-\mathrm{H} \cdots A$ & $D-\mathrm{H}$ & $\mathrm{H} \cdots A$ & $D \cdots A$ & $D-\mathrm{H} \cdots A$ \\
\hline $\mathrm{C} 3-\mathrm{H} 3 \cdots \mathrm{N} 5^{\mathrm{i}}$ & 0.93 & 2.55 & $3.459(4)$ & 167 \\
$\mathrm{C} 13-\mathrm{H} 13 \cdots \mathrm{N} 1^{\mathrm{ii}}$ & 0.93 & 2.49 & $3.408(4)$ & 169 \\
\hline
\end{tabular}

Symmetry codes: (i) $x-1, y, z$; (ii) $x, y, z+1$. 